\title{
De novo Biosynthesis of Odd-Chain Fatty Acids in Yarrowia lipolytica Enabled by Modular Pathway Engineering
}

\author{
Young-kyoung Park ${ }^{1 *}$, Rodrigo Ledesma-Amaro ${ }^{2 *}$ and Jean-Marc Nicaud ${ }^{1}$ \\ ${ }^{1}$ Université Paris-Saclay, INRAE, AgroParisTech, Micalis Institute, Jouy-en-Josas, France, ${ }^{2}$ Imperial College Centre for \\ Synthetic Biology and Department of Bioengineering, Imperial College London, London, United Kingdom
}

OPEN ACCESS

Edited by:

Fayza Daboussi,

Institut National de la Recherche

Agronomique (INRA), France

Reviewed by:

Jiazhang Lian,

Zhejiang University, China

Carol Sze Ki Lin,

City University of Hong Kong,

Hong Kong

*Correspondence:

Young-kyoung Park

youngkyoung.park@inra.fr

Rodrigo Ledesma-Amaro

r.ledesma-amaro@imperial.ac.uk

Specialty section:

This article was submitted to

Synthetic Biology,

a section of the journal

Frontiers in Bioengineering and

Biotechnology

Received: 31 August 2019 Accepted: 27 December 2019

Published: 22 January 2020

Citation:

Park Y, Ledesma-Amaro R and Nicaud J-M (2020) De novo Biosynthesis of

Odd-Chain Fatty Acids in Yarrowia lipolytica Enabled by Modular

Pathway Engineering.

Front. Bioeng. Biotechnol. 7:484.

doi: 10.3389/fbioe.2019.00484
Microbial oils are regarded as promising alternatives to fossil fuels as concerns over environmental issues and energy production systems continue to mount. Odd-chain fatty acids (FAs) are a type of valuable lipid with various applications: they can serve as biomarkers, intermediates in the production of flavor and fragrance compounds, fuels, and plasticizers. Microorganisms naturally produce FAs, but such FAs are primarily even-chain; only negligible amounts of odd-chain FAs are generated. As a result, studies using microorganisms to produce odd-chain FAs have had limited success. Here, our objective was to biosynthesize odd-chain FAs de novo in Yarrowia lipolytica using inexpensive carbon sources, namely glucose, without any propionate supplementation. To achieve this goal, we constructed a modular metabolic pathway containing seven genes. In the engineered strain expressing this pathway, the percentage of odd-chain FAs out of total FAs was higher than in the control strain (3.86 vs. 0.84\%). When this pathway was transferred into an obese strain, which had been engineered to accumulate large amounts of lipids, odd-chain fatty acid production was 7.2 times greater than in the control ( 0.05 vs. $0.36 \mathrm{~g} / \mathrm{L})$. This study shows that metabolic engineering research is making progress toward obtaining efficient cell factories that produce odd-chain FAs.

\footnotetext{
Keywords: odd-chain fatty acids, propionyl-CoA, Yarrowia lipolytica, metabolic engineering, Golden Gate assembly, synthetic biology
}

\section{INTRODUCTION}

Microbial oils (lipids and fatty acid-derived products) are regarded as promising alternatives to fossil fuels in the face of growing concerns over environmental issues and energy production. To lessen the cost of producing microbial oils, considerable effort has been dedicated to enhancing production yield (Dulermo and Nicaud, 2011; Tai and Stephanopoulos, 2013; Qiao et al., 2015); using low-cost carbon substrates (Papanikolaou et al., 2013; Lazar et al., 2014; Guo et al., 2015; Ledesma-Amaro and Nicaud, 2016); and targeting high-value lipids (Xue et al., 2013; Xie et al., 2015). Odd-chain fatty acids (FAs), a type of valuable lipid, are a product with potential because they can be used in a variety of applications. Notably, research has revealed that odd-chain FAs with chain lengths of 15 and 17 carbons may have functional importance for nutrition and medical field. For example, cis-9-heptadecenoic acid has anti-inflammatory effects and can help treat psoriasis, allergies, and autoimmune diseases (Degwert et al., 1998). Pentadecanoic acid and heptadecanoic 
acid can be used as biomarkers of food intake in dietary assessments, the risk of coronary heart disease (CHD), and the risk of type II diabetes mellitus (Forouhi et al., 2014; Jenkins et al., 2015; Pedersen et al., 2016; Pfeuffer and Jaudszus, 2016). The chemical properties and potential biological activities of odd-chain FAs are now being more extensively studied (Rezanka and Sigler, 2009), so it is possible that novel nutritional and pharmaceutical applications could soon be discovered. In addition, odd-chain FAs and their derivatives are precursors for manufacturing substances such as pesticides, flavor and fragrance compounds, hydraulic fluids, plasticizers, coatings, and other industrial chemicals (Fitton and Goa, 1991; Avis, 2000; Clausen et al., 2010; Köckritz et al., 2010). Despite the broad range of applications for FAs, studies aiming to produce odd-chain FAs using microorganisms have had limited success because microorganisms produce a much greater proportion of evenchain FAs than odd-chain FAs.

Generally, de novo fatty acid synthesis in microorganisms begins with the condensation of acetyl-CoA and malonylCoA (Figure 1A). Then, the elongation step occurs: longchain FAs are synthesized in a reaction catalyzed by fatty acid synthase (FAS). The resulting acyl-CoA products are esterified to generate lysophosphatidic acid (LPA), then phosphatidic acid (PA), and finally diacylglycerol (DAG) before forming triacylglycerol (TAG), the compound in which the lipids are stored. For odd-chain FAs, propionyl-CoA could be converted from propionate is a primer for the fatty acid synthesis. The condensation of both propionyl-CoA and malonyl-CoA results in the formation of 3-oxovaleryl-ACP, which is the launching point for odd-chain FA synthesis. This five-carbon compound goes through elongation, where two carbons are added in each cycle. Then odd-chain FAs can be synthesized as described in Figure 1B. However, most microorganisms require the presence of propionate in the medium to produce odd-chain FAs. Wu and San have shown that E. coli can produce odd-chain FAs - namely undecanoic acid (C11:0), tridecanoic acid (C13:0), and pentadecanoic acid (C15:0)-if grown with propionatesupplemented medium (Wu and San, 2014). They introduced a propionyl-CoA synthetase ( $p r p E$ ) from Salmonella enterica and acyl-ACP thioesterases (TEs) from Umbellularia californica and
Ricinus communis. Additionally, propionate supplementation has allowed various yeasts (e.g., Yarrowia lipolytica, Rhodotorula glutinis, Cryptococcus curvatus, and Kluyveromyces polysporus) to produce odd-chain FAs (Zheng et al., 2012; Kolouchová et al., 2015). More recently, in Y. lipolytica, metabolic engineering and the optimization of propionate feeding helped boost the production of odd-chain FAs, namely heptadecenoic acid (C17:1) (Park et al., 2018).

Therefore, to date, most studies have been focused on processes that involve propionate supplementation. However, due to the high cost (Poirier et al., 1995; Aldor et al., 2002) and toxic effects of propionate (Fontanille et al., 2012; Park and Nicaud, 2019), it is crucial to find alternative pathways for generating propionyl-CoA to be able to produce odd-chain FAs on large scales. There have been a few studies in which odd-chain FAs have been produced using glucose. Tseng and Prather have shown that, in E. coli, the production of very short odd-chain FAs (i.e., propionate, trans-2-pentenoate, and valerate) can be increased through the upregulation of threonine biosynthesis (Tseng and Prather, 2012). Another study demonstrated that the overexpression of threonine biosynthesis in E. coli resulted in increased levels of odd-chain FAs (mainly C15:0): from 0.006 to $0.246 \mathrm{~g} / \mathrm{L}$. This study further modified the experimental strain by replacing the native $\beta$-ketoacyl-ACP synthase (encoded by $\mathrm{FabH}$ ) with one from Bacillus subtilis (encoded by FabHI) so that there was a biochemical preference for propionyl-CoA over acetyl-CoA (Lee et al., 2013). However, to date, no one has reported the de novo production of odd-chain FAs in Y. lipolytica or in any other yeasts without propionate supplementation. Consequently, we need more research on metabolic engineering approaches for producing odd-chain FAs in this group of microorganisms.

Of the several microbial hosts used in such systems, the oleaginous yeast $Y$. lipolytica is the most studied and has been engineered to produce large amounts of lipids and lipid derivatives, such as ricinoleic acid (Beopoulos et al., 2014), conjugated linoleic acids (Imatoukene et al., 2017), cyclopropane FAs (Czerwiec et al., 2019), and cocoa butter-like oils (Papanikolaou et al., 2003). Y. lipolytica can naturally grow in a broad range of substrates and has been further engineered to use even more substrates (Ledesma-Amaro et al., 2016). In addition,



B

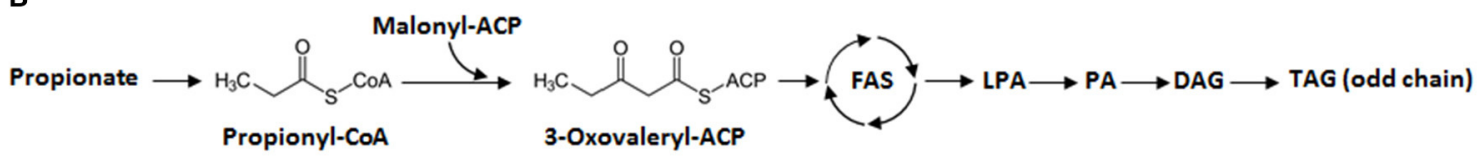

FIGURE 1 | Lipid synthesis in Y. lipolytica. (A) Synthesis of even-chain fatty acids (FAs) from glucose. (B) Synthesis of odd-chain FAs from propionate. First, there is elongation of fatty acyl-CoA by fatty acid synthase (FAS). Second, the resulting even- or odd-chain fatty acyl-CoA is transformed into lysophosphatidic acid (LPA), phosphatidic acid (PA), and diacylglycerol (DAG), in that order, before finally forming triacylglycerol (TAG). 
many different synthetic biology tools have been created for and applied in Y. lipolytica, such as Gibson assembly, Golden Gate assembly, TALEN editing, and CRISPR/Cas9 editing [please see the recent review by Larroude et al. (2018) for more details]. These tools used in tandem with genetic and metabolic engineering strategies have boosted the capacities of Y. lipolytica, making the yeast into a promising host for biotechnological production processes.

The objective of this study was to biosynthesize odd-chain FAs de novo from glucose without propionate supplementation. We constructed a modular metabolic pathway for synthesizing propionyl-CoA from oxaloacetate in Y. lipolytica and confirmed that accumulation of odd-chain FAs was increased. We also investigated whether it could be competitive to produce oddchain FAs from propionyl-CoA via the methylcitrate pathway using the engineered strain. Additionally, we overexpressed the pyruvate dehydrogenase (PDH) complex in the cytosol to see if it could improve the conversion of $\alpha$-ketobutyrate to propionylCoA. This work demonstrates that our metabolic engineering strategy for directing metabolic fluxes through specific pathways can enhance odd-chain FA production.

\section{MATERIALS AND METHODS}

\section{Strains and Media}

Media and growth conditions for E. coli were previously described by Sambrook and Russell (2001); those for Y. lipolytica were previously described by Barth and Gaillardin (1997). Rich medium (YPD) and minimal glucose medium (YNB) were prepared as described elsewhere (Milckova et al., 2004). The YNB contained $0.17 \%(\mathrm{w} / \mathrm{v})$ yeast nitrogen base (without amino acids and ammonium sulfate, YNBww), 0.5\% (w/v) $\mathrm{NH}_{4} \mathrm{Cl}$, $50 \mathrm{mM} \mathrm{KH} 2 \mathrm{PO}_{4}-\mathrm{Na}_{2} \mathrm{HPO}_{4}(\mathrm{pH} 6.8$ ), and 2\% (w/v) glucose. To complement strain auxotrophies, $0.1 \mathrm{~g} / \mathrm{L}$ of uracil or leucine was added as necessary. To screen for hygromycin resistance, $250 \mu \mathrm{g} / \mathrm{ml}$ of hygromycin was added to the YPD. Solid media were prepared by adding $1.5 \%(\mathrm{w} / \mathrm{v})$ agar.

\section{Construction of Plasmids and Strains (E. coli and Y. lipolytica)}

We used standard molecular genetic techniques (Sambrook and Russell, 2001). Restriction enzymes were obtained from New England Biolabs (Ipswich, MA, USA). PCR amplification was performed in an Eppendorf 2720 Thermal Cycler with either Q5 High-Fidelity DNA Polymerase (New England Biolabs) or GoTaq DNA polymerases (Progema, WI, USA). PCR fragments were purified using a PCR Purification Kit (Macherey-Nagel, Duren, Germany), and plasmids were purified with a Plasmid Miniprep Kit (Macherey-Nagel).

The plasmids used in this study were constructed using Golden Gate assembly, as described in Celinska et al. (2017). The genes in the $\mathrm{A}, \mathrm{T}$, and $\mathrm{H}$ module were obtained via PCR using the genomic DNA of Y. lipolytica W29. Internal BsaI recognition sites were removed via PCR using the primers listed in Supplementary Table 1 . The plasmids for each module included the Zeta sequence, the URA3 ex marker, and gene expression cassettes containing the TEF1 promoter and LIP2 terminator.
For the cytosolic PDH complex, all the genes were synthesized and cloned in the plasmid pUC57 by GenScript Biotech (New Jersey, US). Cytosolic PDX1 was cloned into the expression plasmid (JME2563) using the BamHI and AvrII restriction sites. The other four genes were cloned into two plasmids (JME4774 and JME4775) using Golden Gate assembly.

To disrupt $P H D 1$, the cassettes were constructed to include a promoter ( $p P H D 1)$, a marker (URA3 or LEU2), and a terminator (TPHD1), which allowed the ORF gene to be removed via homologous recombination, as described in Papanikolaou et al. (2013).

Gene expression and disruption cassettes were prepared by NotI digestion and transformed into $Y$. lipolytica strains using the lithium acetate method, as described previously (Barth and Gaillardin, 1997). Gene integration and disruption were verified via colony PCR using the primers listed in Supplementary Table 1 . The replicative plasmid harboring the Cre gene (JME547; Table 1) was used for marker rescue (Fickers et al., 2003). After transformation with the Cre expression plasmid, the loss of the marker gene was verified on YNB with/without uracil. The loss of the replicative plasmid was checked using replica plating on YPD with/without hygromycin after culturing on YPD for $24 \mathrm{~h}$. To construct the prototrophic strain, a LEU2 fragment from plasmid JMP2563 was transformed. All the strains and plasmids used in this study are listed in Table 1.

\section{Culture Conditions for the Lipid Biosynthesis Experiments}

The lipid biosynthesis experiments were carried out in minimal media, and the cultures were prepared as follows: an initial preculture was established by inoculating $10 \mathrm{~mL}$ of YPD medium in $50 \mathrm{~mL}$ Erlenmeyer flasks. Then, the pre-culture was incubated overnight at $28^{\circ} \mathrm{C}$ and $180 \mathrm{rpm}$. The resulting cell suspension was washed with sterile distilled water and used to inoculate $50 \mathrm{~mL}$ of minimal medium YNBD6 containing $0.17 \%(\mathrm{w} / \mathrm{v})$ yeast nitrogen base (without amino acids and ammonium sulfate, YNBww, Difco), $0.15 \%$ (w/v) $\mathrm{NH}_{4} \mathrm{Cl}, 50 \mathrm{mM} \mathrm{KH} \mathrm{PO}_{4}-\mathrm{Na}_{2} \mathrm{HPO}_{4}$ buffer ( $\mathrm{pH} 6.8$ ), and 6\% (w/v) glucose. This medium had been placed in $250 \mathrm{~mL}$ Erlenmeyer flasks. The cultures were then incubated at $28^{\circ} \mathrm{C}$ and $180 \mathrm{rpm}$.

\section{Lipid Determination}

Lipids were extracted from 10 to $20 \mathrm{mg}$ of freeze-dried cells and converted into FA methyl esters (FAMEs) using the procedure described by Browse et al. (1986). The FAMEs were then analyzed using gas chromatography (GC), which was carried out with a Varian 3900 instrument equipped with a flame ionization detector and a Varian FactorFour vf-23ms column, where the bleed specification at $260^{\circ} \mathrm{C}$ is $3 \mathrm{pA}(30 \mathrm{~m}, 0.25 \mathrm{~mm}, 0.25 \mu \mathrm{m})$. The FAMEs were identified via comparisons with commercial standards (FAME32, Supelco) and quantified using the internal standard method, which involves the addition of $100 \mu \mathrm{g}$ of commercial dodecanoic acid (Sigma-Aldrich). Commercial oddchain FAs (Odd Carbon Straight Chains Kit containing 9 FAs, OC9, Supelco) were converted into their FAMEs using the same method employed with the yeast samples. They were then 
TABLE 1 | The plasmids and strains used in this study.

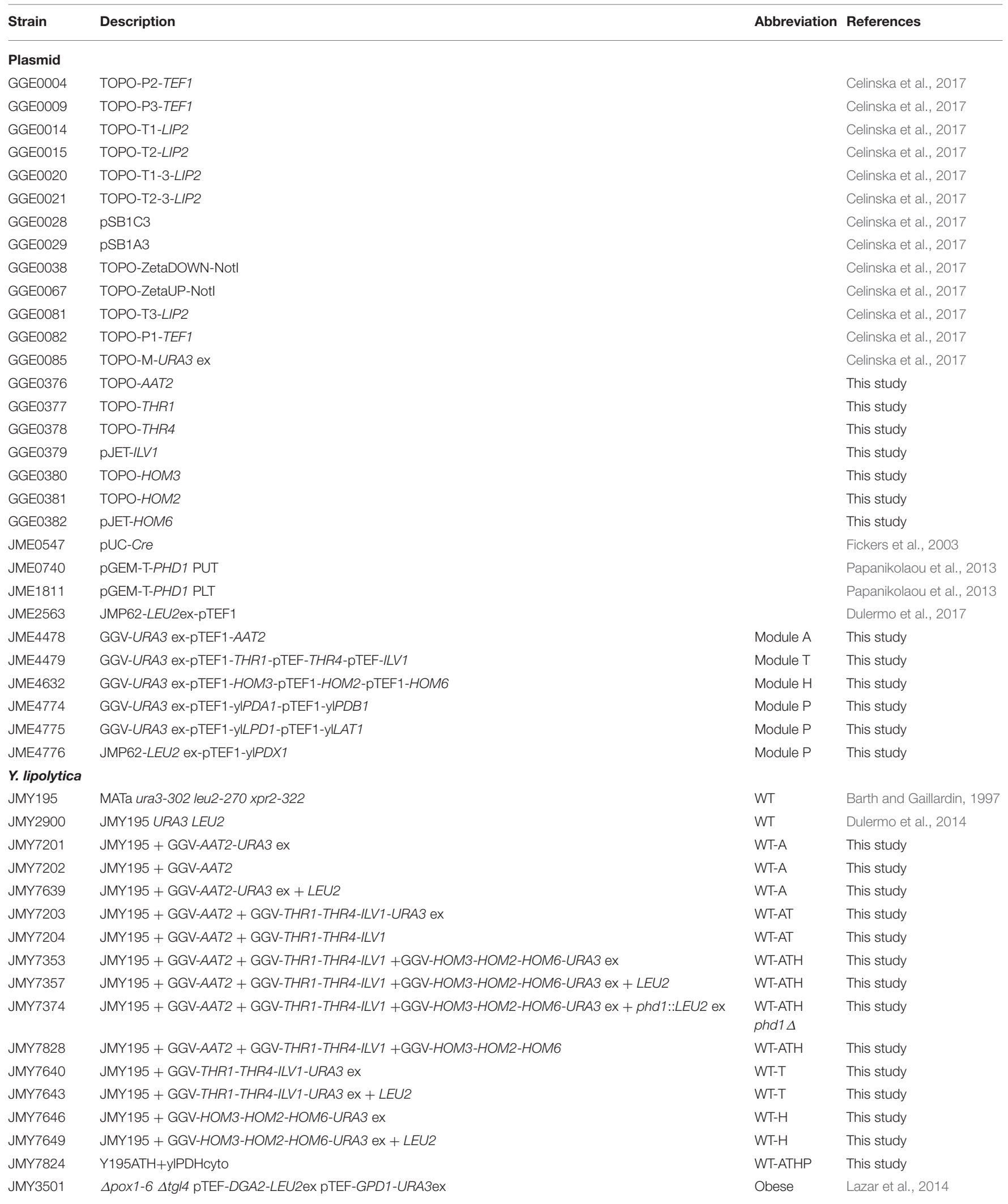


TABLE 1 | Continued

\begin{tabular}{|c|c|c|c|}
\hline Strain & Description & Abbreviation & References \\
\hline JMY3820 & $\Delta p o x 1-6 \Delta t g 14$ pTEF-DGA2 pTEF-GPD1 & Obese & Lazar et al., 2014 \\
\hline JMY7206 & JMY3820 + GGV-AAT2-URA3 ex & Obese-A & This study \\
\hline JMY7207 & JMY3820 + GGV-AAT2 & Obese-A & This study \\
\hline JMY7208 & JMY3820 + GGV-AAT2 + GGV-THR1-THR4-ILV1-URA3 ex & Obese-AT & This study \\
\hline JMY7267 & JMY3820 + GGV-AAT2 + GGV-THR1-THR4-ILV1 & Obese-AT & This study \\
\hline JMY7412 & MY3820 + GGV-AAT2 + GGV-THR1-THR4-ILV1 + GGV-HOM3-HOM2-HOM6-URA3 ex + LEU2 & Obese-ATH & This study \\
\hline JMY7413 & MY3820 + GGV-AAT2 + GGV-THR1-THR4-ILV1 + GGV-HOM3-HOM2-HOM6 & Obese-ATH & This study \\
\hline JMY7414 & JMY7413 + phd1::LEU2 ex & $\begin{array}{l}\text { Obese-ATH } \\
\text { phd1 } \Delta\end{array}$ & This study \\
\hline JMY7417 & JMY7413 + phd1::LEU2 ex + URA3 & $\begin{array}{l}\text { Obese-ATH } \\
\text { phd1 } \Delta\end{array}$ & This study \\
\hline JMY7826 & Y3820ATH+ylPDHcyto & Obese-ATHP & This study \\
\hline
\end{tabular}

identified using GC and compared with the odd-chain FAs from the yeast samples.

To determine dry cell weight (DCW), $2 \mathrm{~mL}$ of the culture was taken from the flasks, washed, and lysophilized in a pre-weighed tube. The differences in mass corresponded to the mg of cells found in $2 \mathrm{~mL}$ of culture.

\section{RESULTS}

\section{Modular Pathway Engineering Was Used for Odd-Chain Fatty Acid Synthesis}

Propionyl-CoA is a key primer in the synthesis of odd-chain FAs. It can be synthesized using $\beta$-oxidation from direct precursors, propionate, or long-chain FAs. It can also be created from other metabolites via several metabolic pathways, such as the citramalate/2-ketobutyrate pathway, the aspartate/2ketobutyrate pathway, the methylmalonyl-CoA pathway, the 3-hydroxypropionate pathway, and the isoleucine or valine degradation pathway (Supplementary Figure 1; Han et al., 2013; Lee et al., 2013). Here, we tested if the overexpression of the $\alpha$-ketobutyrate pathway-which produces threonine as an intermediate-could increase levels of propionyl-CoA in Y. lipolytica. As shown in Figure 2A, the pathway eventually forms the amino acids aspartate, homoserine, and threonine from oxaloacetate. Then, threonine is deaminated to generate $\alpha$-ketobutyrate, a reaction catalyzed by threonine dehydratase. Alpha-ketobutyrate is directly or sequentially converted into propionyl-CoA by the pyruvate dehydrogenase (PDH) complex or pyruvate oxidase, respectively. The upregulation of threonine has previously been used to boost propionyl-CoA availability in E. coli. Lee et al. showed that levels of oddchain FAs could be increased by introducing the threonine biosynthesis pathway (which creates $\alpha$-ketobutyrate from aspartate semialdehyde), especially when mutated homoserine dehydrogenase (thrA*, reduced feedback inhibition) was also expressed (Lee et al., 2013). The percentage of oddchain FAs out of total FAs increased from $<1$ to $18 \%$ by overexpressing the threonine pathway in E. coli. The predominant odd-chain FA produced was pentadecanoic acid (C15:0).

In this study, we enhanced the extended threonine biosynthesis pathway (the aspartate/ $\alpha$-ketobutyrate pathway) from oxaloacetate to $\alpha$-ketobutyrate-by overexpressing seven genes (Figure 2A). There were three modules (Figures 2A,C): the aspartate synthesis module (module A), which included AAT2; the homoserine synthesis module (module $\mathrm{H}$ ), which included HOM3, HOM2, and HOM6; and the threonine synthesis module (module T), which included THR1, THR4, and ILV1. While threonine and $\alpha$-ketobutyrate (module T) are synthesized in the mitochondria in S. cerevisiae, the same may not be true in Y. lipolytica. While the locations of the relevant enzymes are as yet unknown in Y. lipolytica, predictive analyses (Supplementary Table 4) suggest enzyme location may differ between S. cerevisiae and Y. lipolytica. Because the module $\mathrm{T}$ enzymes were predicted to occur in the cytoplasm in Y. lipolytica, we used the original sequence of each gene in this study, as described in Supplementary Table 3. However, more research is needed to confirm enzyme locations in $Y$. lipolytica. The genes in each module were cloned into one plasmid using Golden Gate assembly (Figure 2B). They were expressed under the constitutive promoter pTEF1, and the expression cassette of each module was randomly integrated into the genome. Each module $(\mathrm{A}, \mathrm{T}$, and $\mathrm{H})$ in the pathway was overexpressed in Y. lipolytica both individually and in tandem. The strain with the full modular pathway (ATH) was constructed by removing and reusing the URA3 marker (Supplementary Figure 2). We verified gene integration using colony PCR with the primer set of promoters and the ORF gene (Supplementary Table 1).

\section{The Engineered Strain Could Produce Odd-Chain Fatty Acids Using Glucose as Its Sole Carbon Source}

To determine whether the modular metabolic pathway was effective in producing odd-chain FAs, we evaluated the performance of the engineered strains overexpressing the individual modules and the entire pathway. The strains 


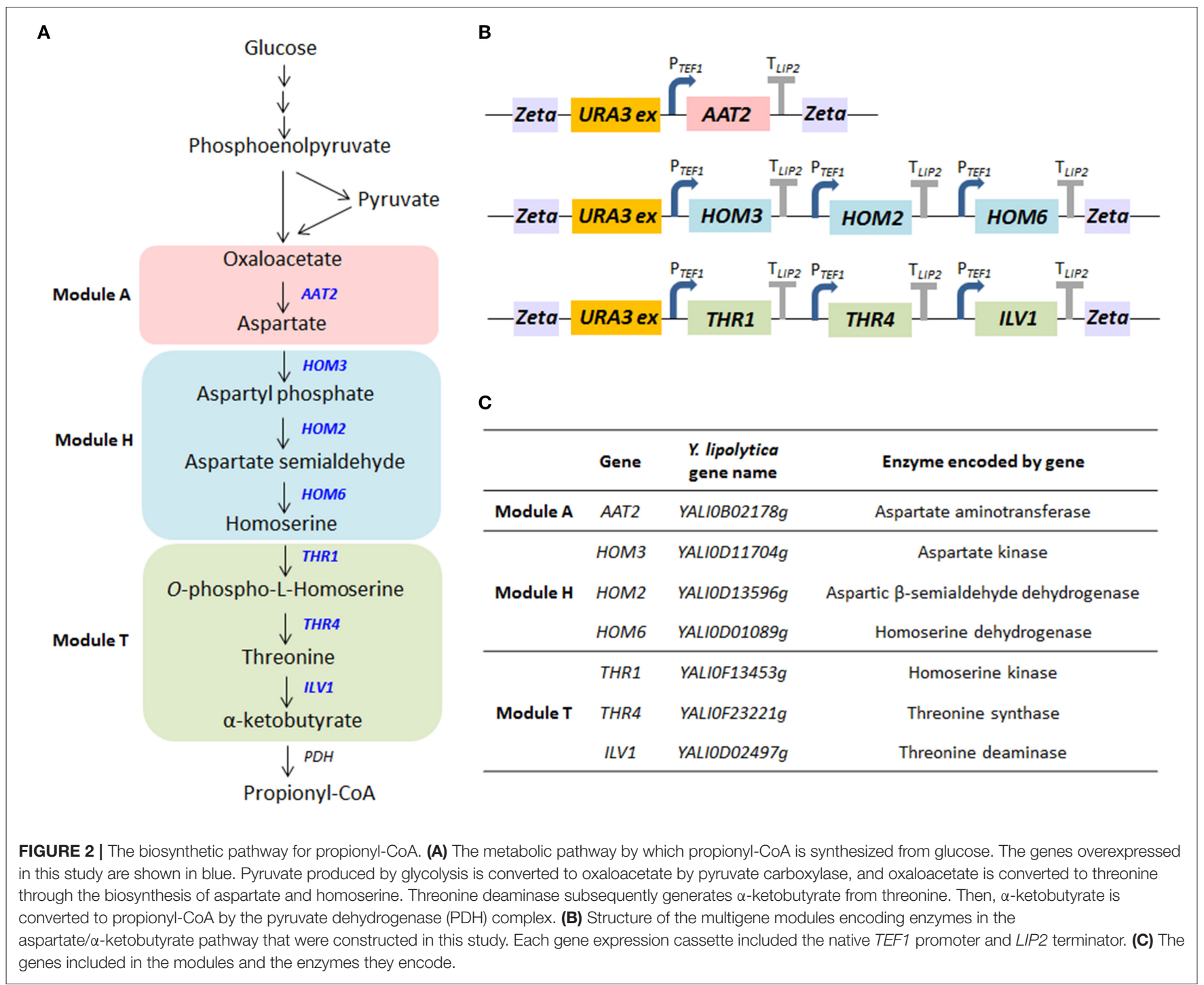

were cultivated in YNBD6 medium under nitrogen limitation conditions $(\mathrm{C} / \mathrm{N}=60)$, which have been found to positively influence lipid synthesis (Beopoulos et al., 2012; LedesmaAmaro et al., 2016). For the WT-A strain, which overexpressed $A A T 2$, and the WT-T strain, which overexpressed THR1, THR4, and $I L V 1$, total lipid content (\%, g/g DCW) was lower than in the wild-type (WT) strain; the percentage of odd-chain FAs out of total FAs was similar (Table 2). For the WT$\mathrm{H}$ strain, which overexpressed HOM3, HOM2, and HOM6, this percentage was slightly greater $(1.91 \%)$ than that in the WT strain $(0.84 \%)$ (Table 2 and Figure $3 \mathrm{~A}$ ). For the WTATH strain, which overexpressed the entire pathway, oddchain FA synthesis was significantly greater, odd-chain FA content $(\%, \mathrm{~g} / \mathrm{g}$ DCW) was 3.8 times higher, and odd-chain FA titers $(\mathrm{g} / \mathrm{L})$ were 3.6 times higher than in the WT strain; the percentage of odd-chain FAs out of total FAs was $3.86 \%$, which was 4.6 times higher than the value seen in the WT strain. These results indicate that the engineered aspartate/ $\alpha$ ketobutyrate pathway can supply propionyl-CoA; they also show that the full pathway is needed for effective odd-chain FA synthesis.

WT-ATH primarily produced C17:1 FAs (Figure 3B). This profile resembles that of an engineered $Y$. lipolytica strain that received propionate supplementation (Park et al., 2018). This finding implies that enhancing carbon flux through the $\alpha$-ketobutyrate pathway can boost propionyl-CoA availability and odd-chain FA synthesis the same way that propionate supplementation can.

\section{Odd-Chain Fatty Acid Production Was Significantly Improved in a Lipid-Accumulating Strain}

Once we had determined that the strain overexpressing the full modular pathway produced more odd-chain FAs, we wanted to boost their accumulation. Previously, we had engineered a Y. lipolytica strain, JMY3501, to accumulate large amounts of lipids (Lazar et al., 2014). We eliminated lipid degradation and 
TABLE 2 | Fatty acid (FA) production in the wild-type (WT) strain and the engineered strains after growth on YNBD6 medium for $120 \mathrm{~h}$.

\begin{tabular}{|c|c|c|c|c|c|c|}
\hline \multirow[t]{2}{*}{ Strain } & \multirow[t]{2}{*}{ DCW (g/L) } & \multicolumn{2}{|c|}{ Lipid content \% } & \multirow[t]{2}{*}{ Odd-chain FA /Total FA (\%) } & \multicolumn{2}{|c|}{ Lipid titer (g/L) } \\
\hline & & Total FA & Odd-chain FA & & Total FA & Odd-chain FA \\
\hline WT & $18.65 \pm 0.15$ & $19.13 \pm 2.22$ & $0.16 \pm 0.00$ & $0.84 \pm 0.09$ & $3.571 \pm 0.442$ & $0.029 \pm 0.000$ \\
\hline WT-A & $17.30 \pm 0.15$ & $16.60 \pm 1.05$ & $0.14 \pm 0.01$ & $0.87 \pm 0.03$ & $2.873 \pm 0.207$ & $0.025 \pm 0.003$ \\
\hline WT-T & $16.50 \pm 0.00$ & $14.41 \pm 0.21$ & $0.15 \pm 0.00$ & $1.03 \pm 0.01$ & $2.378 \pm 0.035$ & $0.024 \pm 0.001$ \\
\hline WT-H & $16.30 \pm 0.55$ & $10.96 \pm 0.09$ & $0.21 \pm 0.03$ & $1.91 \pm 0.21$ & $1.788 \pm 0.075$ & $0.034 \pm 0.005$ \\
\hline WT-ATH & $16.90 \pm 0.25$ & $15.73 \pm 0.95$ & $0.61 \pm 0.13$ & $3.86 \pm 0.57$ & $2.656 \pm 0.121$ & $0.103 \pm 0.020$ \\
\hline
\end{tabular}

The values represent the means and standard deviations for two independent experiments. DCW, dry cell weight.

A

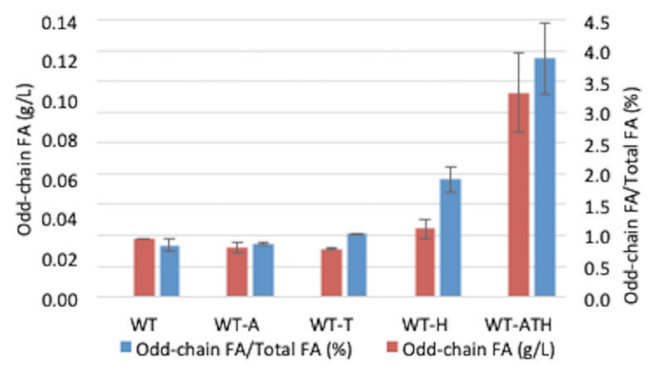

B

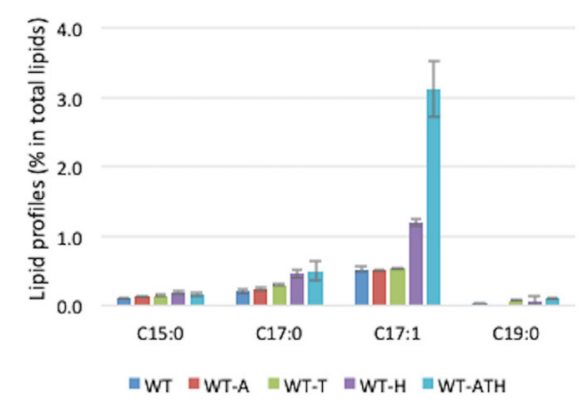

FIGURE 3 | Odd-chain fatty acid production and profiles for the wild-type and engineered strains. (A) Odd-chain fatty acid (FA) titers (g/L) and the percentage of odd-chain FAs relative to total FAs in the wild-type (WT) and engineered strains. (B) Odd-chain FA profiles (percentage of each FA out of total FAs) of the WT strain and engineered strains. WT, control strain; WT-A, strain expressing module A; WT-T, strain expressing module T; WT-H, strain expressing module H; WT-ATH, strain expressing the full pathway (all three modules). The results represent the means and standard deviations for two independent experiments.

remobilization in this obese strain by deleting the POX (POX16) genes (Beopoulos et al., 2008) as well as the TGL4 gene, which encodes a triglyceride lipase (Dulermo et al., 2013). In addition, we overexpressed DGA2, which encodes the major acyl-CoA:diacylglycerol acyltransferase (Beopoulos et al., 2012), and GPD1, which encodes glycerol-3-phosphate dehydrogenase, in order to push and pull TAG biosynthesis (Dulermo and Nicaud, 2011; Tai and Stephanopoulos, 2013). Therefore, using JMY3501, we built a new obese strain that overexpressed our full modular pathway. It was called the obese-ATH strain. We then studied lipid production under the same conditions as before. As expected, total lipid accumulation was 2.29fold greater in the obese-ATH strain than in the WT-ATH strain (Tables 2, 3). Interestingly, the obese-ATH strain also accumulated more odd-chain FAs: the percentage of odd-chain FAs out of total FAs was $5.64 \%$ in the obese-ATH strain vs. $3.86 \%$ in the WT-ATH strain. The obese-ATH strain produced $0.36 \mathrm{~g} / \mathrm{L}$ of odd-chain FAs, which is 7.2 times greater than the amount produced by the regular obese strain. The obeseATH strain and the WT-ATH strain differed in their evenchain FA profiles (Table 4). The obese-ATH had slightly higher levels of C16:0 and slightly lower levels of C18:1, a common pattern seen in strains with the obese background regardless of the carbon source (Lazar et al., 2014; Ledesma-Amaro et al., 2016).

\section{The Disruption of PHD1 in the Methylcitrate Cycle Did Not Increase Odd-Chain Fatty Acid Production}

In a previous study, we inactivated $P H D 1$, the gene that encodes 2-methylcitrate dehydratase, which catalyzes the conversion of 2-methyl citrate to 2-methyl-cis-aconitate in the methylcitrate cycle; we showed that the resulting higher levels of propionylCoA could be used to synthesize greater amounts of odd-chain FAs (Park et al., 2018). To investigate whether the inhibition of the methylcitrate cycle-via the deletion of PHD1-could further improve the accumulation of odd-chain FAs, we disrupted the PHD1 gene in both the WT-ATH strain and the obese-ATH strain. The two phd $1 \Delta$ strains displayed higher total lipid content compared to their relative controls (Supplementary Table 2), a result that was also demonstrated in Papanikolaou et al. (2013); however, they also displayed lower percentages of odd-chain FAs out of total FAs (Figure 4). This latter negative effect was significantly more pronounced in the obese-ATH phd $1 \Delta$ strain than in the WT-ATH phd1 $\Delta$ strain. For the obese-ATH phd1 $\Delta$ strain, this figure dropped by $50 \%$, and levels of odd-chain FAs were $67 \%$ of those seen in the relative control $(0.24$ vs. $0.36 \mathrm{~g} / \mathrm{L}$ ). These results suggest that disrupting the methylcitrate cycle does not provide the benefits seen previously (Park et al., 2018) when strains are already overexpressing the aspartate/ $\alpha$ ketobutyrate pathway. 
TABLE 3 | Fatty acid (FA) production in the obese strain and the obese-ATH strain after growth on YNBD6 medium for $120 \mathrm{~h}$.

\begin{tabular}{|c|c|c|c|c|c|c|}
\hline \multirow[t]{2}{*}{ Strain } & \multirow[t]{2}{*}{$\mathrm{DCW}(\mathrm{g} / \mathrm{L})$} & \multicolumn{2}{|c|}{ Lipid content $\%$} & \multirow[t]{2}{*}{ Odd-chain FA /Total FA (\%) } & \multicolumn{2}{|c|}{ Lipid titer (g/L) } \\
\hline & & Total FA & Odd-chain FA & & Total FA & Odd-chain FA \\
\hline Obese & $19.20 \pm 0.04$ & $37.11 \pm 0.14$ & $0.25 \pm 0.00$ & $0.68 \pm 0.01$ & $7.125 \pm 0.012$ & $0.049 \pm 0.001$ \\
\hline Obese-ATH & $17.62 \pm 0.03$ & $36.02 \pm 0.39$ & $2.03 \pm 0.05$ & $5.64 \pm 0.06$ & $6.347 \pm 0.058$ & $0.358 \pm 0.009$ \\
\hline
\end{tabular}

The values represent the means and standard deviations for two independent experiments. DCW, dry cell weight.

TABLE 4 | Comparison of the lipid profiles (\% of each FA) of the WT-ATH strain and the obese-ATH strain.

\begin{tabular}{|c|c|c|c|c|c|c|c|c|c|}
\hline Strain & C15:0 & C16:0 & C16:1 & C17:0 & C17:1 & C18:0 & C18:1 & C18:2 & C19:0 \\
\hline WT-ATH & $0.16 \pm 0.03$ & $8.31 \pm 0.75$ & $6.13 \pm 0.00$ & $0.50 \pm 0.14$ & $3.11 \pm 0.40$ & $5.96 \pm 0.68$ & $61.31 \pm 2.43$ & $11.02 \pm 0.26$ & $0.10 \pm 0.00$ \\
\hline Obese-ATH & $0.40 \pm 0.00$ & $13.07 \pm 0.01$ & $7.25 \pm 0.11$ & $0.76 \pm 0.00$ & $4.26 \pm 0.06$ & $5.00 \pm 0.17$ & $50.12 \pm 0.27$ & $14.11 \pm 0.16$ & $0.22 \pm 0.13$ \\
\hline
\end{tabular}

The values represent the means and standard deviations for two independent experiments.

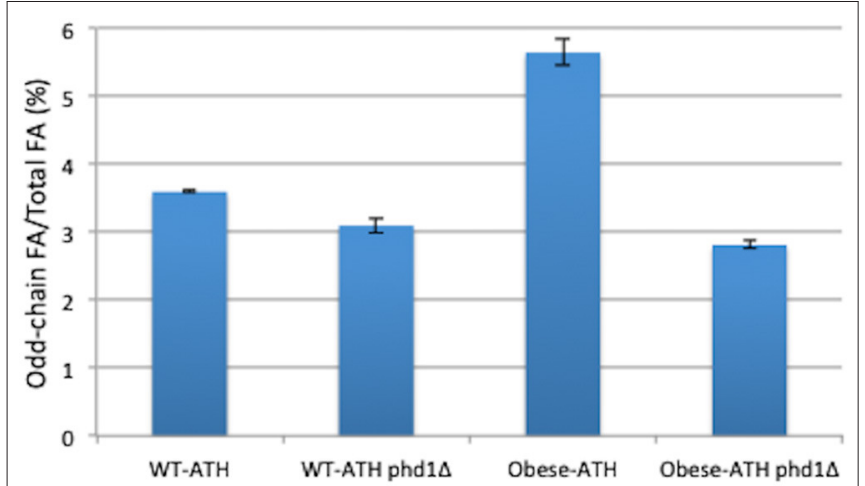

FIGURE 4 | Odd-chain fatty acid production in the PHD1-disrupted strains. The percentage of odd-chain fatty acids (FAs) relative to total FAs in the PHD1-disrupted strains and their respective controls. The results represent the means and standard deviations for two independent experiments.

The action of 2-methylcitrate dehydratase is restricted to the mitochondria. Consequently, disabling this enzyme does not directly improve cytosolic levels of propionyl-CoA. In the previous studies showing that the disruption of PHD1 boosted odd-chain FA synthesis (Papanikolaou et al., 2013; Park et al., 2018), there was also propionate supplementation. Taken together, these findings suggest that, when the methylcitrate cycle is disrupted, the overexpression of the aspartate/ $\alpha$-ketobutyrate pathway has a weaker effect on propionyl-CoA levels than does propionate supplementation.

\section{Overexpression of the Cytosolic Pyruvate Dehydrogenase (PDH) Complex Improved Lipid Synthesis but Reduced Odd-Chain Fatty Acid Production}

The PDH complex consists of three main catalytic components: E1 (pyruvate dehydrogenase, encoded by PDA1 and PDB1), E2 (dihydrolipoamide acetyltransferase, encoded by LAT1), and E3 (dihydrolipoamide dehydrogenase, encoded by LPD1). There is a fourth component (protein $\mathrm{X}$, encoded by PDX1) that binds to and positions E3 relative to E2. In Y. lipolytica, the $\mathrm{PDH}$ complex is found in mitochondria and catalyzes the pathway from pyruvate to acetyl-CoA. A few studies have examined the functional expression of the PDH complex in Y. lipolytica. One study attempted to overexpress the direct pathway from pyruvate to acetyl-CoA in a coordinated manner (Markham et al., 2018), and another study showed that the individual overexpression of PDA1 (shared subunits with $\alpha$-ketoglutarate dehydrogenase) improved $\alpha$-ketoglutarate production (Guo et al., 2014).

Here, we wanted to redirect the aspartate/ $\alpha$-ketobutyrate pathway to produce propionyl-CoA via the PDH complex, which has already been shown to be possible in E. coli (Danchin et al., 1984; Tseng and Prather, 2012; Lee et al., 2013). The PDH complex was built so it could be associated with the full modular pathway (ATH) and could provide a pool of propionyl-CoA in the cytosol where lipid synthesis takes place. The mitochondrial targeting sequences (MTSs) of each gene were predicted using MitoProt (Claros and Vincens, 1996), and the gene sequences used in this study are described in Supplementary Table 3. We then created the obese-ATHP strain by overexpressing the cytosolic PDH subunits in the obese-ATH strain. Next, to explore lipid accumulation dynamics, the strain was grown in YNBD6 with lipoic acid, which is required for cytosolic PDH activity in yeast (Kozak et al., 2014). Compared to the control strain, the obese-ATHP strain had significantly lower levels of oddchain FAs (a 3.8 times lower percentage of odd-chain FAs out of total FAs); however, total lipid levels were higher (Table 5). The increase in lipid production might have resulted from the increased levels of cytosolic acetyl-CoA resulting from the overexpression of the PDH complex. A similar strategy was utilized in Saccharomyces cerevisiae, where PDH was introduced into the cytosol and was found to increase levels of acetyl-CoA (Kozak et al., 2014; Lian et al., 2014) and of the target compound of interest. Since acetyl-CoA is a key precursor in the production of both even- and odd-chain FAs, increasing levels of acetyl-CoA promotes lipid synthesis in general. However, the substantially lower levels of odd-chain FAs in the obese-ATHP strain implies that the $\mathrm{PDH}$ complex shows greater specificity for pyruvate than for $\alpha$-ketobutyrate. The higher $\mathrm{Km}$ value of $\alpha$-ketobutyrate 
TABLE 5 | Fatty acid (FA) production in the obese-ATH strain and the obese-ATHP strain after growth on YNBD6 medium for $120 \mathrm{~h}$.

\begin{tabular}{|c|c|c|c|c|c|c|}
\hline \multirow[t]{2}{*}{ Strain } & \multirow[t]{2}{*}{ DCW (g/L) } & \multicolumn{2}{|c|}{ Lipid content $\%$} & \multirow[t]{2}{*}{ Odd-chain FA /Total FA (\%) } & \multicolumn{2}{|c|}{ Lipid titer (g/L) } \\
\hline & & Total FA & Odd-chain FA & & Total FA & Odd-chain FA \\
\hline Obese-ATH & $15.68 \pm 0.02$ & $29.23 \pm 0.01$ & $1.59 \pm 0.03$ & $5.44 \pm 0.10$ & $4.582 \pm 0.010$ & $0.249 \pm 0.005$ \\
\hline Obese-ATHP & $15.25 \pm 0.25$ & $33.89 \pm 1.06$ & $0.48 \pm 0.03$ & $1.42 \pm 0.03$ & $5.171 \pm 0.246$ & $0.073 \pm 0.005$ \\
\hline
\end{tabular}

The values represent the means and standard deviations for two independent experiments. DCW, dry cell weight.

compared to that of pyruvate has been seen elsewhere, such as in E. coli (Bisswanger, 1981), Neurospora crassa (Harding et al., 1970), and mammalian cells (Bremer, 1969). Therefore, it is important to explore enzyme engineering strategies that modify substrate specificity or that introduce other enzymes that can convert acetyl-CoA to propionyl-CoA with a view to further improving odd-chain FA production via a threoninebased upregulation strategy.

\section{DISCUSSION}

In this study, we developed a synthetic biological strategy for the de novo production of odd-chain FAs in Y. lipolytica. It is important to note that the wild-type $Y$. lipolytica strain produces only negligible amounts of odd-chain FAs even though it has an excellent capacity to accumulate large quantities of lipids. Several studies have shown that propionate supplementation can increase the production of odd-chain FAs (Fontanille et al., 2012; Kolouchová et al., 2015; Park et al., 2018). However, research has yet to explore the de novo production of odd-chain FAs from sugars in Y. lipolytica.

The overexpression of the aspartate/ $\alpha$-ketobutyrate pathway (from oxaloacetate to homoserine and threonine) via Golden Gate assembly resulted in higher levels of odd-chain FAs being produced from glucose. The best strain generated a level of oddchain FAs, $0.36 \mathrm{~g} / \mathrm{L}$ in flask that is the highest to date to be achieved in $Y$. lipolytica without propionate supplementation. Furthermore, it is comparable to the levels seen in a previous study of ours that employed propionate supplementation, where odd-chain FAs titers were 0.14 and $0.57 \mathrm{~g} / \mathrm{L}$ in the wild-type strain and the obese strain, respectively (Park et al., 2018). To further increase the amount of propionyl-CoA produced via the overexpression of threonine synthesis, we constructed a cytosolic pyruvate dehydrogenase (PDH) complex. Because of the lower specificity of the PDH complex for $\alpha$-ketobutyrate vs. pyruvate, the engineered strain generated lower levels of propionyl-CoA than did the relative control; however, the increased levels of acetyl-CoA in the engineered strain led to larger amounts of total FAs. This study is the first to describe the functional expression of the native $\mathrm{PDH}$ complex in the cytosol in $Y$. lipolytica, an approach that could also be employed to produce acetyl-CoA-derived compounds, such as polyhydroxybutyrates, isoprenoids, sterols, polyketides, polyphenols, alkanes, and alkenes (Nielsen, 2014).
Before the approach described here can be applied at industrial scales, certain issues must be resolved. It is necessary to perform research in which combinatorial pathway analysis (Lütke-Eversloh and Stephanopoulos, 2008), targetedproteomics analysis (Redding-Johanson et al., 2011), and genome-scale metabolic network modeling (Xu et al., 2011) are used to identify bottlenecks and enhance metabolic fluxes to propionyl-CoA and odd-chain FAs. The results of the $\mathrm{PDH}$ overexpression experiment suggest that acetyl-CoA is a competitive precursor to propionyl-CoA in odd-chain FA synthesis and is also a precursor in lipid synthesis. Therefore, better balancing the pools of acetyl-CoA and propionyl-CoA could be key in further increasing odd-chain FA content. One way to improve odd-chain FA synthesis is to introduce enzymes-such as CoA transferase-that redirect CoA moieties from acetyl-CoA to propionyl-CoA (Yang et al., 2012) or that have greater specificity for 3-oxovaleryl-ACP than for acetoacetyl-CoA (Slater et al., 1998). Overall, this work has shown that applying synthetic biological engineering strategies in Y. lipolytica to improve odd-chain FA production could be useful in a wide range of pharmaceutical and industrial contexts.

\section{DATA AVAILABILITY STATEMENT}

The raw data supporting the conclusions of this article will be made available by the authors, without undue reservation, to any qualified researcher.

\section{AUTHOR CONTRIBUTIONS}

YP, RL-A, and J-MN planned the study. YP designed and carried out the experiments and drafted the manuscript. All the authors revised and approved the final manuscript.

\section{FUNDING}

YP was the recipient of a Ph.D. scholarship from the Kwanjeong Educational Foundation (KEF).

\section{SUPPLEMENTARY MATERIAL}

The Supplementary Material for this article can be found online at: https://www.frontiersin.org/articles/10.3389/fbioe. 2019.00484/full\#supplementary-material 


\section{REFERENCES}

Aldor, I. S., Kim, S. W., Jones Prather, K. L., and Keasling, J. D. (2002). Metabolic engineering of a novel propionate-independent pathway for the production of poly(3-hydroxybutyrate-co-3-hydroxyvalerate) in recombinant Salmonella enterica serovar typhimurium. Appl. Environ. Microbiol. 68, 3848-3854. doi: 10.1128/AEM.68.8.3848-3854.2002

Avis, T. J. (2000). Synthesis and biological characterization of (Z)-9-heptadecenoic and (Z)-6-methyl-9-heptadecenoic acids: fatty acids with antibiotic activity produced by Pseudozyma flocculosa. J. Chem. Ecol. 26, 987-1000. doi: 10.1023/A:1005464326573

Barth, G., and Gaillardin, C. (1997). Physiology and genetics of the dimorphic fungus Yarrowia lipolytica. FEMS Microbiol. Rev. 19, 219-237. doi: 10.1111/j.1574-6976.1997.tb00299.x

Beopoulos, A., Haddouche, R., Kabran, P., Dulermo, T., Chardot, T., and Nicaud, J. M. (2012). Identification and characterization of DGA2, an acyltransferase of the DGAT1 acyl-CoA:diacylglycerol acyltransferase family in the oleaginous yeast Yarrowia lipolytica. New insights into the storage lipid metabolism of oleaginous yeasts. Appl. Microbiol. Biotechnol. 93, 1523-1537. doi: 10.1007/s00253-011-3506-x

Beopoulos, A., Mrozova, Z., Thevenieau, F., Le Dall, M. T., Hapala, I., Papanikolaou, S., et al. (2008). Control of lipid accumulation in the yeast Yarrowia lipolytica. Appl. Environ. Microbiol. 74, 7779-7789. doi: 10.1128/AEM.01412-08

Beopoulos, A., Verbeke, J., Bordes, F., Guicherd, M., Bressy, M., Marty, A., et al. (2014). Metabolic engineering for ricinoleic acid production in the oleaginous yeast Yarrowia lipolytica. Appl. Microbiol. Biotechnol. 98, 251-262. doi: 10.1007/s00253-013-5295-x

Bisswanger, H. (1981). Substrate specificity of the pyruvate dehydrogenase complex from Escherichia coli. J. Biol. Chem. 256, 815-822.

Bremer, J. (1969). Pyruvate dehydrogenase, substrate specificity and product inhibition. Eur. J. Biochem. 8, 535-540. doi: 10.1111/j.1432-1033.1969.tb00559.x

Browse, J., McCourt, P. J., and Somerville, C. R. (1986). Fatty acid composition of leaf lipids determined after combined digestion and fatty acid methyl ester formation from fresh tissue. Anal. Biochem. 152, 141-145. doi: 10.1016/0003-2697(86)90132-6

Celinska, E., Ledesma-Amaro, R., Larroude, M., Rossignol, T., Pauthenier, C., and Nicaud, J. M. (2017). Golden Gate Assembly system dedicated to complex pathway manipulation in Yarrowia lipolytica. Microb. Biotechnol. 10, 450-455. doi: 10.1111/1751-7915.12605

Claros, M. G., and Vincens, P. (1996). Computational method to predict mitochondrially imported proteins and their targeting sequences. Eur. J. Biochem. doi: 10.1111/j.1432-1033.1996.00779.x

Clausen, C. A., Coleman, R. D., and Yang, V. W. (2010). Fatty acid-based formulations for wood protection against mold and sapstain. For. Prod. J. 60, 301-304. doi: 10.13073/0015-7473-60.3.301

Czerwiec, Q., Idrissitaghki, A., Imatoukene, N., Nonus, M., Thomasset, B., Nicaud, J. M., et al. (2019). Optimization of cyclopropane fatty acids production in Yarrowia lipolytica. Yeast 36, 143-151. doi: 10.1002/yea.3379

Danchin, A., Dondon, L., and Daniel, J. (1984). Metabolic alterations mediated by 2-ketobutyrate in Escherichia coli K12. MGG Mol. Gen. Genet. 193, 473-478. doi: 10.1007/BF00382086

Degwert, J., Jacob, J., and Steckel, F., Inventors. (1998). Use of Cis-9-Heptadecenoic Acid for trEating Psoriasis and Allergies. Hamburg: Beiersdorf AG.

Dulermo, R., Brunel, F., Dulermo, T., Ledesma-Amaro, R., Vion, J., Trassaert, M., et al. (2017). Using a vector pool containing variable-strength promoters to optimize protein production in Yarrowia lipolytica. Microb. Cell Fact. 16, 1-11. doi: 10.1186/s12934-017-0647-3

Dulermo, R., Gamboa-Meléndez, H., Dulermo, T., Thevenieau, F., and Nicaud, J. M. (2014). The fatty acid transport protein Fatlp is involved in the export of fatty acids from lipid bodies in Yarrowia lipolytica. FEMS Yeast Res. 14, 883-896. doi: 10.1111/1567-1364.12177

Dulermo, T., and Nicaud, J. M. (2011). Involvement of the G3P shuttle and $\beta$ oxidation pathway in the control of TAG synthesis and lipid accumulation in Yarrowia lipolytica. Metab. Eng. 13, 482-491. doi: 10.1016/j.ymben.2011.05.002

Dulermo, T., Tréton, B., Beopoulos, A., Gnankon, A. P. K., Haddouche, R., and Nicaud, J. M. (2013). Characterization of the two intracellular lipases of $Y$. lipolytica encoded by TGL3 and TGL4 genes: new insights into the role of intracellular lipases and lipid body organisation. Biochim. Biophys. Acta. 1831, 1486-1495. doi: 10.1016/j.bbalip.2013.07.001

Fickers, P., Le Dall, M. T., Gaillardin, C., Thonart, P., and Nicaud, J. M. (2003). New disruption cassettes for rapid gene disruption and marker rescue in the yeast Yarrowia lipolytica. J. Microbiol. Methods 55, 727-737. doi: 10.1016/j.mimet.2003.07.003

Fitton, A., and Goa, K. L. (1991). Azelaic acid: a review of its pharmacological properties and therapeutic efficacy in acne and hyperpigmentary skin disorders. Drugs 41, 780-798. doi: 10.2165/00003495-199141050-00007

Fontanille, P., Kumar, V., Christophe, G., Nouaille, R., and Larroche, C. (2012). Bioconversion of volatile fatty acids into lipids by the oleaginous yeast Yarrowia lipolytica. Bioresour. Technol. 114, 443-449. doi: 10.1016/j.biortech.2012.02.091

Forouhi, N. G., Koulman, A., Sharp, S. J., Imamura, F., Kröger, J., Schulze, M. B., et al. (2014). Differences in the prospective association between individual plasma phospholipid saturated fatty acids and incident type 2 diabetes: the EPIC-InterAct case-cohort study. Lancet Diabetes Endocrinol. 2, 810-818. doi: 10.1016/S2213-8587(14)70146-9

Guo, H., Madzak, C., Du, G., Zhou, J., and Chen, J. (2014). Effects of pyruvate dehydrogenase subunits overexpression on the $\alpha$-ketoglutarate production in Yarrowia lipolytica WSH-Z06. Appl. Microbiol. Biotechnol. 98, 7003-7012. doi: 10.1007/s00253-014-5745-0

Guo, Z., Duquesne, S., Bozonnet, S., Cioci, G., Nicaud, J. M., Marty, A., et al. (2015). Development of cellobiose-degrading ability in Yarrowia lipolytica strain by overexpression of endogenous genes. Biotechnol. Biofuels 8, 1-16. doi: 10.1186/s13068-015-0289-9

Han, J., Hou, J., Zhang, F., Ai, G., Li, M., Cai, S., et al. (2013). Multiple propionyl coenzyme a-supplying pathways for production of the bioplastic poly(3-Hydroxybutyrate-co-3-Hydroxyvalerate) in Haloferax mediterranei. Appl. Environ. Microbiol. 79, 2922-2931. doi: 10.1128/AEM.03915-12

Harding, R. W., Caroline, D. F., and Wagner, R. P. (1970). The pyruvate dehydrogenase fraction complex from the mitochondrial of Neurospora crassa. Arch. Biochem. Biophys. 138, 653-661. doi: 10.1016/0003-9861(70)90393-0

Imatoukene, N., Verbeke, J., Beopoulos, A., Idrissi Taghki, A., Thomasset, B., Sarde, C. O., et al. (2017). A metabolic engineering strategy for producing conjugated linoleic acids using the oleaginous yeast Yarrowia lipolytica. Appl. Microbiol. Biotechnol. 101, 4605-4616. doi: 10.1007/s00253-017-8240-6

Jenkins, B., West, J. A., and Koulman, A. (2015). A review of odd-chain fatty acid metabolism and the role of pentadecanoic acid (C15:0) and heptadecanoic acid (C17:0) in health and disease. Molecules 20, 2425-2444. doi: $10.3390 /$ molecules 20022425

Köckritz, A., Blumenstein, M., and Martin, A. (2010). Catalytic cleavage of methyl oleate or oleic acid. Eur. J. Lipid Sci. Technol. 112, 58-63. doi: 10.1002/ejlt.200900103

Kolouchová, I., Schreiberová, O., Sigler, K., Masák, J., and Rezanka, T. (2015). Biotransformation of volatile fatty acids by oleaginous and non-oleaginous yeast species. FEMS Yeast Res. 15, 1-8. doi: 10.1093/femsyr/fov076

Kozak, B. U., van Rossum, H. M., Luttik, M. A. H., Akeroyd, M., Benjamin, K. R., Wu, L., et al. (2014). Engineering acetyl coenzyme A supply: functional expression of a bacterial pyruvate dehydrogenase complex in the cytosol of Saccharomyces cerevisiae. mBio 5, 1-11. doi: 10.1128/mBio.01696-14

Larroude, M., Rossignol, T., Nicaud, J. M., and Ledesma-Amaro, R. (2018). Synthetic biology tools for engineering Yarrowia lipolytica. Biotechnol. Adv. 36, 2150-2164. doi: 10.1016/j.biotechadv.2018.10.004

Lazar, Z., Dulermo, T., Neuvéglise, C., Crutz-Le Coq, A. M., and Nicaud, J. M. (2014). Hexokinase-A limiting factor in lipid production from fructose in Yarrowia lipolytica. Metab. Eng. 26, 89-99. doi: 10.1016/j.ymben.2014. 09.008

Ledesma-Amaro, R., Lazar, Z., Rakicka, M., Guo, Z., Fouchard, F., Coq, A. M. C., et al. (2016). Metabolic engineering of Yarrowia lipolytica to produce chemicals and fuels from xylose. Metab. Eng. 38, 115-124. doi: 10.1016/j.ymben.2016.07.001

Ledesma-Amaro, R., and Nicaud, J. M. (2016). Metabolic engineering for expanding the substrate range of Yarrowia lipolytica. Trends Biotechnol. 34, 798-809. doi: 10.1016/j.tibtech.2016.04.010

Lee, G. J., Haliburton, J. R., Hu, Z., and Schirmer, A. W., Inventors. (2013). Production of Odd Chain Fatty Acid Derivatives in Recombinant Microbial Cells. San Francisco, CA: LS9, INC. 
Lian, J., Si, T., Nair, N. U., and Zhao, H. (2014). Design and construction of acetylCoA overproducing Saccharomyces cerevisiae strains. Metab. Eng. 24, 139-149. doi: 10.1016/j.ymben.2014.05.010

Lütke-Eversloh, T., and Stephanopoulos, G. (2008). Combinatorial pathway analysis for improved L-tyrosine production in Escherichia coli: identification of enzymatic bottlenecks by systematic gene overexpression. Metab. Eng. 10, 69-77. doi: 10.1016/j.ymben.2007.12.001

Markham, K. A., Palmer, C. M., Chwatko, M., Wagner, J. M., Murray, C., Vazquez, S., et al. (2018). Rewiring Yarrowia lipolytica toward triacetic acid lactone for materials generation. Proc. Natl. Acad. Sci. U. S. A 115, 2096-2101. doi: 10.1073/pnas.1721203115

Milckova, K., Roux, E., Athenstaedt, K., Andrea, S., Daum, G., Chardot, T., et al. (2004). Lipid accumulation, lipid body formation, and acyl coenzyme a oxidases of the yeast Yarrowia lipolytica. Appl. Environ. Microbiol. 70, 3918-3924. doi: 10.1128/AEM.70.7.3918-3924.2004

Nielsen, J. (2014). Synthetic biology for engineering acetyl coenzyme a metabolism in yeast. $m$ Bio 5, 14-16. doi: 10.1128/mBio.02153-14

Papanikolaou, S., Beopoulos, A., Koletti, A., and Thevenieau, F. (2013). Importance of the methyl-citrate cycle on glycerol metabolism in the yeast Yarrowia lipolytica. J. Biotechnol. 168, 303-314. doi: 10.1016/j.jbiotec.2013.10.025

Papanikolaou, S., Muniglia, L., Chevalot, I., Aggelis, G., and Marc, I. (2003). Accumulation of a cocoa-butter-like lipid by Yarrowia lipolytica cultivated on agro-industrial residues. Curr. Microbiol. 46, 124-130. doi: 10.1007/s00284-002-3833-3

Park, Y. K., Dulermo, T., Ledesma-Amaro, R., and Nicaud, J. M. (2018). Optimization of odd chain fatty acid production by Yarrowia lipolytica. Biotechnol. Biofuels 11, 1-12. doi: 10.1186/s13068-018-1154-4

Park, Y. K., and Nicaud, J. M. (2019). Screening a genomic library for genes involved in propionate tolerance in Yarrowia lipolytica. Yeast. doi: 10.1002/yea.3431. [Epub ahead of print].

Pedersen, H. K., Gudmundsdottir, V., Nielsen, H. B., Hyotylainen, T., Nielsen, T., Jensen, B. A. H., et al. (2016). Human gut microbes impact host serum metabolome and insulin sensitivity. Nature 535, 376-381. doi: $10.1038 /$ nature 18646

Pfeuffer, M., and Jaudszus, A. (2016). Pentadecanoic and Heptadecanoic acids: multifaceted odd-chain fatty acids. Adv. Nutr. 7, 730-734. doi: 10.3945/an.115.011387

Poirier, Y., Nawrath, C., and Somerville, C. (1995). Production of polyhydroxyalkanoates, a family of biodegradable plastics and elastomers, in Bacteria and Plants. Nat. Biotechnol. 13, 142-150. doi: 10.1038/nbt0 295-142

Qiao, K., Imam Abidi, S. H., Liu, H., Zhang, H., Chakraborty, S., Watson, N., et al. (2015). Engineering lipid overproduction in the oleaginous yeast Yarrowia lipolytica. Metab. Eng. 29, 56-65. doi: 10.1016/j.ymben.2015. 02.005

Redding-Johanson, A. M., Batth, T. S., Chan, R., Krupa, R., Szmidt, H. L., Adams, P. D., et al. (2011). Targeted proteomics for metabolic pathway optimization: application to terpene production. Metab. Eng. 13, 194-203. doi: 10.1016/j.ymben.2010.12.005
Rezanka, T., and Sigler, K. (2009). Odd-numbered very-long-chain fatty acids from the microbial, animal, and plant kingdoms. Pro. Lipid. Res. 48, 206-238. doi: 10.1016/j.plipres.2009.03.003

Sambrook, J., and Russell, D. W. (2001). Molecular Cloning. A Laboratory Manual, $3 r d$ Edn. Cold Spring Harbor, NY:Cold Spring Harbor Laboratory Press.

Slater, S., Houmiel, K. L., Tran, M., Mitsky, T. A., Taylor, N. B., Padgette, S. R., et al. (1998). Multiple $\beta$-ketothiolases mediate $\operatorname{Poly}(\beta$-Hydroxyalkanoate) copolymer synthesis in Ralstonia eutropha. J. Bacteriol. 180, 1979-1987. doi: 10.1128/JB.180.8.1979-1987.1998

Tai, M., and Stephanopoulos, G. (2013). Engineering the push and pull of lipid biosynthesis in oleaginous yeast Yarrowia lipolytica for biofuel production. Metab. Eng. 15, 1-9. doi: 10.1016/j.ymben.2012.08.007

Tseng, H. C., and Prather, K. L. J. (2012). Controlled biosynthesis of odd-chain fuels and chemicals via engineered modular metabolic pathways. Proc. Natl. Acad. Sci. U. S. A 109, 17925-17930. doi: 10.1073/pnas.1209002109

Wu, H., and San, K. Y. (2014). Engineering Escherichia coli for odd straight medium chain free fatty acid production. Appl. Microbiol. Biotechnol. 98, 8145-8154. doi: 10.1007/s00253-014-5882-5

Xie, D., Jackson, E. N., and Zhu, Q. (2015). Sustainable source of omega-3 eicosapentaenoic acid from metabolically engineered Yarrowia lipolytica: from fundamental research to commercial production. Appl. Microbiol. Biotechnol. 99, 1599-1610. doi: 10.1007/s00253-014-6318-y

Xu, P., Ranganathan, S., Fowler, Z. L., Maranas, C. D., and Koffas, M. A. G. (2011) Genome-scale metabolic network modeling results in minimal interventions that cooperatively force carbon flux towards malonyl-CoA. Metab. Eng. 13, 578-587. doi: 10.1016/j.ymben.2011.06.008

Xue, Z., Sharpe, P. L., Hong, S. P., Yadav, N. S., Xie, D., Short, D. R., et al. (2013). Production of omega-3 eicosapentaenoic acid by metabolic engineering of Yarrowia lipolytica. Nat. Biotechnol. 31, 734-740. doi: 10.1038/nbt.2622

Yang, Y., Brigham, C. J., Song, E., Jeon, J., Rha, C. K., and Sinskey, A. J. (2012). Biosynthesis of poly (3-hydroxybutyrate- co-3- hydroxyvalerate) containing a predominant amount of 3-hydroxyvalerate by engineered Escherichia coli expressing propionate-CoA transferase. J. Appl. Microbiol. 13, 815-823. doi: 10.1111/j.1365-2672.2012.05391.x

Zheng, Y., Chi, Z., Ahring, B. K., and Chen, S. (2012). Oleaginous yeast Cryptococcus curvatus for biofuel production: ammonia's effect. Biomass and Bioenergy 37, 114-121. doi: 10.1016/j.biombioe.2011.12.022

Conflict of Interest: The authors declare that the research was conducted in the absence of any commercial or financial relationships that could be construed as a potential conflict of interest.

Copyright (C) 2020 Park, Ledesma-Amaro and Nicaud. This is an open-access article distributed under the terms of the Creative Commons Attribution License (CC BY). The use, distribution or reproduction in other forums is permitted, provided the original author(s) and the copyright owner(s) are credited and that the original publication in this journal is cited, in accordance with accepted academic practice. No use, distribution or reproduction is permitted which does not comply with these terms. 